\title{
Isolation and Identification of the Five Novel Flavonoids from Genipa americana Leaves
}

\author{
Larissa Marina Pereira Silva ${ }^{1}$, Jovelina Samara Ferreira Alves ${ }^{1}$, \\ Emerson Michell da Silva Siqueira ${ }^{1}$, Manoel André de Souza Neto ${ }^{1}$, Lucas Silva Abreu ${ }^{2}$ (D, \\ Josean Fechine Tavares ${ }^{2}$, Dayanne Lopes Porto ${ }^{3}$, Leandro de Santis Ferreira ${ }^{3}$, \\ Daniel Pecoraro Demarque ${ }^{4}\left(\mathbb{D}\right.$, Norberto Peporine Lopes ${ }^{4}\left(\mathbb{D}\right.$, Cícero Flávio Soares Aragão ${ }^{3}(\mathbb{D}$ \\ and Silvana Maria Zucolotto ${ }^{1, *}$ \\ 1 Research Group on Bioactive Natural Products (PNBio), Laboratory of Pharmacognosy, \\ Department of Pharmacy, Federal University of Rio Grande do Norte (UFRN), Natal 59010-180, Brazil; \\ larissamarinaps@gmail.com (L.M.P.S.); jsfa.farma@gmail.com (J.S.F.A.); \\ siqueira_emerson@hotmail.com (E.M.d.S.S.); rufinim@gmail.com (M.A.d.S.N.) \\ 2 Multiuser Laboratory of Characterization and Analysis (LMCA), Federal University of Paraíba, \\ João Pessoa 58051-900, Brazil; lucas.abreu@ltf.ufpb.br (L.S.A.); josean@ltf.ufpb.br (J.F.T.) \\ 3 Laboratory of Quality Control of Medications (LCQMed), Department of Pharmacy, \\ Federal University of Rio Grande do Norte (UFRN), Natal 59010-180, Brazil; \\ daylopesporto@hotmail.com (D.L.P.); lean_sf@yahoo.com.br(L.d.S.F.); \\ cicero.aragao@yahoo.com.br (C.F.S.A.) \\ 4 Nucleus Research in Natural and Synthetic Products (NPPNS), Department of Physics and Chemistry, \\ Faculty of Pharmaceutical Sciences of Ribeirão Preto, University of São Paulo, São Paulo 14040-903, Brazil; \\ dpdemarque@gmail.com (D.P.D.); npelopes@fcfrp.usp.br (N.P.L.) \\ * Correspondence: silvanazucolotto@ufrnet.br; Tel.: +55-084-33429818
}

Received: 15 August 2018; Accepted: 5 September 2018; Published: 2 October 2018

check for updates

\begin{abstract}
Genipa americana is a medicinal plant popularly known as "jenipapo", which occurs in Brazil and belongs to the Rubiaceae family. It is a species widely distributed in the tropical Central and South America, especially in the Cerrado biome. Their leaves and fruits are used as food and popularly in folk medicine to treat anemias, as an antidiarrheal, and anti-syphilitic. Iridoids are the main secondary metabolites described from G. americana, but few studies have been conducted with their leaves. In this study, the aim was to chemical approach for identify the main compounds present at the extract of $G$. americana leaves. The powdered leaves were extracted by maceration with $\mathrm{EtOH}$ : water $(70: 30, v / v)$, following liquid-liquid partition with petroleum ether, chloroform, ethyl acetate and $n$-butanol. A total of 13 compounds were identified. In addition three flavonoids were isolated from the ethyl acetate fraction: quercetin-3-O-robinoside (GAF 1), kaempferol-3-O-robinoside (GAF 2) and isorhamnetin-3-O-robinoside (GAF 3) and, from $n$-butanol fraction more two flavonoids were isolated, kaempferol-3-O-robinoside-7-O-rhamnoside (robinin) (GAF 4) and isorhamnetin-3-O-robinoside-7-rhamnoside (GAF 5). Chemical structures of these five flavonoids were elucidated using spectroscopic methods (MS, ${ }^{1} \mathrm{H}$ and ${ }^{13} \mathrm{C}-\mathrm{NMR} 1 \mathrm{D}$ and $\left.2 \mathrm{D}\right)$. These flavonoids glycosides were described for the first time in G. americana.
\end{abstract}

Keywords: Rubiaceae; jenipapo; HPLC-ESI-IT-MS/MS; flavonoids glycosides

\section{Introduction}

Genipa americana L. belongs to Rubiaceae family, which contains approximately 140 genera and 2120 species occurring and in various places around the world, mainly in tropical and subtropical regions [1,2]. Genipa americana $\mathrm{L}$. is a native Brazilian plant, with perennial foliage and widely 
distributed in tropical Central and South America, including the Cerrado biome [3-5]. It is popularly known as "jenipapo", which comes from the indigenous language Tupi-Guarani and means "fruit used for painting" [6]. Its fruits are usually processed for liqueurs, candies, and ice cream preparations [7], and are used as tonic against anemia [8]. Ethnopharmacological studies report that different parts of the species have been used in folk medicine, including leaves as an antidiarrheal and anti-syphilitic (decoction) [9]; leaf preparation for treatment of fevers (macerated) [10]; and to treat liver diseases [8].

Most of the phytochemical and pharmacological studies reported to G. americana were conducted with its fruits [4]. Regarding pharmacological studies especially with its leaves there are reports about anti-inflammatory [11], antiangiogenic [12], antidiarrheal, and anti-syphilis activity [9], inhibition of larval development and eclosion of sheep gastrointestinal nematodes [13], antidiabetic [14], and anticonvulsant effects [15].

Concerning the phytochemical composition, it has been reported to G. americana leaves the presence of secondary metabolites such as tannins [13], monoterpenes [16], and flavonoids [17], being iridoids the main secondary metabolites described until this moment [4,16,18-21]. Mainly for the leaves, the presence of following iridoids was already described: geniposidic acid [22], genipatriol [23], genamesides A-D [19], and our group recently isolated and identified the iridoids 1-hydroxy-7-(hydroxymethyl)-1,4aH,5H,7aH-cyclopenta[c]pyran-4-carbaldehyde, and 7-(hydroxymethyl)-1-methoxy-1 $H, 4 \mathrm{a} H, 5 H, 7 \mathrm{a} H$-cyclopenta[c]pyran-4-carbaldehyde [24].

Meanwhile, although flavonoids are a large class of plant secondary metabolites widely distributed throughout the plant kingdom [24], that is structurally resembling in that most have a $C_{15}$ phenyl-benzopyrone skeleton [25], the reports about its presence in G. americana are scarce, making it a topic that needs further investigation. Our research group detected the presence of flavonoids for the first time in G. americana leaves through a fingerprint by thin layer chromatography (TLC) using a specific reagent to detect flavonoids (NP reagent) [24].

In this context, the present study aimed to study the hydroethanolic extract (HE) obtained from leaves of Genipa americana, in terms of its phytochemical composition, especially with respect to flavonoid profiles obtained by UHPLC-DAD and LC-IT-ESI-MS/MS.

\section{Results and Discussion}

\subsection{Extraction and TLC Analysis}

Despite being a class of secondary metabolites that have shown promising biological activities in various studies [26], only our study described the presence of flavonoids in G. americana [24]. Therefore, the phytochemical investigation of this work was conducted to isolate and purify the flavonoids of G. americana leaf extract. Initially, the extract (HE) was fractioned and TLC analysis of the HE and fractions, with specific spray reagents, indicated the presence of flavonoids and iridoids $[7,27]$. The phytochemical screening of the HE, revealed with vanillin sulfuric acid, showed characteristics yellow spots $\left(R_{f}=0.33\right.$ until 0.90$)$, suggesting the presence of flavonoids as observed in our previous work [24]. Afterwards, a liquid-liquid extraction was carried out, affording the following fractions: petroleum ether $(\mathrm{PE})$, chloroform $\left(\mathrm{CHCl}_{3}\right)$, ethyl acetate $(\mathrm{EtOAc}), n$-butanol $(n-\mathrm{BuOH})$ and the residual aqueous fraction (RA). The PE fraction showed no spot in the TLC plate after spraying with vanillin sulfuric acid; $\mathrm{CHCl}_{3}$ fraction showed two purple spots $\left(R_{f}=0.93\right.$ and 0.83$)$, suggesting the presence of iridoids; the EtOAc fraction showed only one zone more evident $\left(R_{f}=0.81\right.$-purple), that indicated the presence of iridoids. When EtOAc fraction was revealed with Natural Product (NP) Reagent (UV $365 \mathrm{~nm})$ a yellow zone was observed $\left(R_{f}=0.55\right)$, suggesting the presence of flavonoids. TLC analysis of the $n$-BuOH fraction showed four yellow and orange spots $\left(R_{f}=0.21\right.$ until $0.50-\mathrm{NP}$ Reagent/UV $365 \mathrm{~nm})$, suggestive of flavonoids [8]. 


\subsection{UHPLC-DAD Characterization}

The Research Group on Bioactive Natural Products (PNBio) has been studying the leaves of G. americana and in a recent publication [24] the presence of two new iridoids for its leaves has been identified, showing the chemical potential of the species.

As described earlier, most of the studies have described the presence of iridoids in the genus Genipa, being considered a chemotaxonomic marker [2,24]. Only our study identified flavonoids in leaves [24]. Furthermore, only one article described the presence of leucoanthocyanidins, catechins, and flavanones in the fruits of G. americana by HPLC analysis [28], and another study described its presence in fruits by colorimetric methods [13], demonstrating that the presence of flavonoids in leaf extract was not described previously.

Extract and fractions were analyzed by ultra-performance liquid chromatography (UHPLC). The chromatogram of the HE, recorded in $254 \mathrm{~nm}$, showed four main peaks (3.5 min, UV: 246, 265 and $350 \mathrm{~nm} ; 3.9 \mathrm{~min}, \mathrm{UV}: 255$ and $354 \mathrm{~nm} ; 16.6 \mathrm{~min}, \mathrm{UV}: 242$ and $287 \mathrm{~nm} ; 17.2 \mathrm{~min}, \mathrm{UV}: 243 \mathrm{~nm})$. Accordingly, the UV spectra of each peak observed in the chromatogram can suggest the presence the flavonoids and iridoids in HE of G. americana leaves (Figure 1).

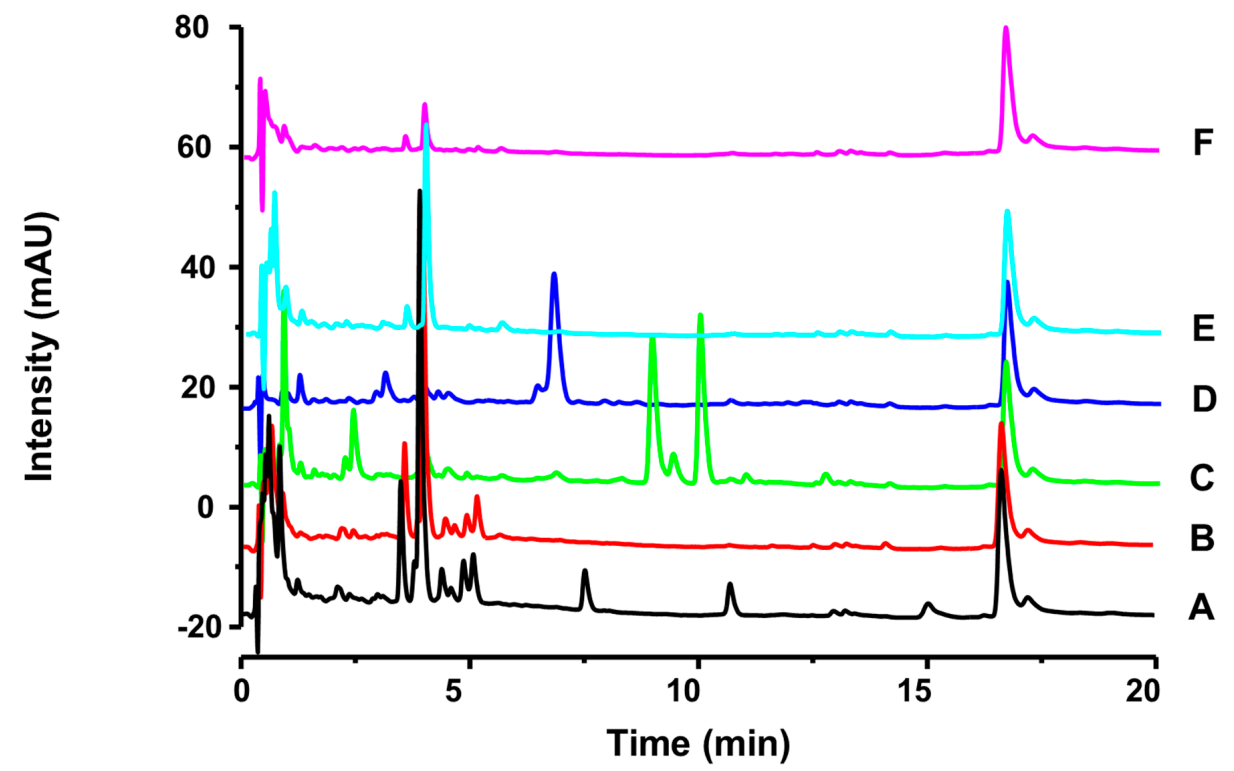

Figure 1. UHPLC-DAD chromatogram of G. americana leaf extract and fractions. Solvent system: mix of acetonitrile: water (with $0.3 \%$ of acetic acid); Flow rate: $0.3 \mathrm{~mL} / \mathrm{min}$; Software LC Solution; Wavelength: $254 \mathrm{~nm}$. column $\mathrm{C}_{18}$ Shim-pack XR-ODS (Shimadzu ${ }^{\circledR}$, Japan) $(30 \times 2 \mathrm{~mm}, 2.2 \mu \mathrm{m})$, the temperature $25 \pm 2{ }^{\circ} \mathrm{C}$. A: EtOAc fraction; $\mathbf{B}: n$-BuOH fraction; C: $\mathrm{CHCl}_{3}$ fraction; D: PE fraction; E: Aqueous residual fraction; F: Extract HE.

\subsection{HPLC-ESI-IT-MS/MS Characterization}

Through the TLC and UHPLC-DAD, we can suggest the presence of iridoids and flavonoids in leaves extract from G. americana. Therefore, it was decided to perform an HPLC-MS/MS study to allow the identification these compounds.

In this way, we selected of HE, Fr.EtOAc, and Fr.n-BuOH to analyze. The HPLC-MS/MS revealed the presence of several majority peaks and allowed suggest the structures of 13 compounds-including five flavonoids (subsequently isolated), using the program DataAnalysis 4.2 (Bruker, Billerica, MA, USA) (Table 1). 
Table 1. Phytochemical profile of leaf extract from G. americana obtained by HPLC-IT-ESI-MS/MS. For conditions, please see Section 3.4.

\begin{tabular}{|c|c|c|c|c|c|c|c|}
\hline \multirow{2}{*}{ Peak n ${ }^{\circ}$} & \multirow{2}{*}{$R t(\min )$} & $m / z$ & $m / z \mathrm{MS}^{2}$ & $m / z \mathrm{MS}^{3}$ & LC-DAD & \multirow{2}{*}{$\begin{array}{l}\text { Molecular } \\
\text { Formula }\end{array}$} & \multirow{2}{*}{ Compound (MM) } \\
\hline & & Pos/Neg & Pos/Neg & Pos/Neg & $\lambda_{\max }[\mathrm{nm}]$ & & \\
\hline 1 & $5.2^{\mathrm{a}}$ & $-/ 342$ & $-/ 179$ & $-/-$ & - & $\mathrm{C}_{16} \mathrm{H}_{22} \mathrm{O}_{8}$ & Coniferin (342) \\
\hline 2 & $24^{\mathrm{b}}$ & $559 / 535,571^{-}$ & $-/ 535$ & 373,210 & - & $\mathrm{C}_{22} \mathrm{H}_{32} \mathrm{O}_{15}$ & Asystasioside D (536) \\
\hline 3 & $25.1^{\mathrm{a}}$ & $397 / 373,409$ & $379,217 / 210$ & $172 / 166,148,122$ & - & $\mathrm{C}_{16} \mathrm{H}_{22} \mathrm{O}_{10}$ & Geniposidic acid (374) \\
\hline 4 & $26.1^{\mathrm{a}}$ & $-/ 357,393$ & $-/ 194$ & $-/-$ & - & $\mathrm{C}_{16} \mathrm{H}_{25} \mathrm{O}_{9}$ & Tarenoside (358) \\
\hline 5 & $26.9^{\mathrm{b}}$ & $-/ 375$ & $-/ 213$ & $-/ 169,125$ & - & $\mathrm{C}_{16} \mathrm{H}_{24} \mathrm{O}_{10}$ & Loganic acid (376) \\
\hline 6 & $33.4^{\mathrm{a}, \mathrm{b}}$ & $355 / 353$ & $162 / 190$ & $-/-$ & & $\mathrm{C}_{16} \mathrm{H}_{18} \mathrm{O}_{9}$ & Chlorogenic acid (354) \\
\hline 7 & $48.8^{\mathrm{a}, \mathrm{b}, \mathrm{c}}$ & $741 / 739^{-}$ & $595 / 593$ & $433,287 / 285$ & 266,346 & $\mathrm{C}_{33} \mathrm{H}_{39} \mathrm{O}_{20}$ & Kaempferol-3-O-hexoside-deoxyhexoside-7-O-deoxyhexoside (740) \\
\hline 8 & $50.1^{\mathrm{a}, \mathrm{b}}$ & $771 / 769$ & $625,463 / 623$ & $317 / 315$ & 255,354 & $\mathrm{C}_{34} \mathrm{H}_{42} \mathrm{O}_{21}$ & Isorhamnetin-3-O-hexoside-deoxyhexoside-7-O-deoxyhexoside (770) \\
\hline 9 & $51.4^{\mathrm{a}, \mathrm{b}}$ & $611 / 609^{-}$ & $465 / 301$ & 303/- & 255,366 & $\mathrm{C}_{27} \mathrm{H}_{30} \mathrm{O}_{16}$ & Quercetin-3-O-hexoside-deoxyhexoside (610) \\
\hline 10 & $51.7^{\mathrm{b}}$ & $-/ 515$ & $-/ 353,190,178$ & $-/-$ & - & $\mathrm{C}_{25} \mathrm{H}_{24} \mathrm{O}_{12}$ & 1,3-Di-O-caffeoylquinic acid (516) \\
\hline 11 & 52 & $519,373,211 / 517,371^{-}$ & $-/-$ & $-/-$ & - & $\mathrm{C}_{22} \mathrm{H}_{30} \mathrm{O}_{14}$ & Teneoside A (518) \\
\hline 12 & $53.9^{\mathrm{a}, \mathrm{b}}$ & $595 / 593$ & $2834.7 / 449,288$ & $-/-$ & 265,355 & $\mathrm{C}_{27} \mathrm{H}_{30} \mathrm{O}_{16}$ & Kaempferol-3-O-hexoside-deoxyhexoside (594) \\
\hline 13 & $55.2^{\mathrm{a}, \mathrm{b}}$ & $625 / 623$ & $479 / 315$ & $317 /-$ & 255,370 & $\mathrm{C}_{28} \mathrm{H}_{33} \mathrm{O}_{16}$ & Isorhamnetin-3-O-hexoside-deoxyhexoside (624) \\
\hline
\end{tabular}

${ }^{\mathrm{a}}=\mathrm{HE} ;{ }^{\mathrm{b}}=$ Fr. EtOAc; ${ }^{\mathrm{c}}=$ Fr. $n$-BuOH; Rt: retention time; MM: molecular mass. 
Coniferin $\left(\mathrm{MM}=342, \mathrm{C}_{16} \mathrm{H}_{22} \mathrm{O}_{8}\right.$, Table 1), showed signals at $342[\mathrm{M}-\mathrm{H}]^{-} ; 179\left[\mathrm{M}-\mathrm{H}-\mathrm{Glc}^{-}\right.$. Already identified in Ginkgo biloba L. (Ginkgoaceae) [29].

Asystasioside $D\left(\mathrm{MM}=536, \mathrm{C}_{22} \mathrm{H}_{32} \mathrm{O}_{15}\right.$, Table 1): showed signals at $559[\mathrm{M}+\mathrm{Na}]^{+}, 535[\mathrm{M}-\mathrm{H}]^{-}$; $571\left[\mathrm{M}+\mathrm{Cl}^{-}, 535,373\right.$ [M - H - Glc]; 210 [M - H - Glc - Glc] [aglycone genipinic acid]. This compound is a iridoid glucoside and had already been described in Asystasia bella (Harv.) [30].

Geniposidic acid $\left(\mathrm{MM}=374, \mathrm{C}_{16} \mathrm{H}_{22} \mathrm{O}_{10}\right.$, Table 1), showed signals at $397[\mathrm{M}+\mathrm{Na}]^{+}, 373[\mathrm{M}-\mathrm{H}]^{-}$, $409[\mathrm{M}+\mathrm{Cl}]^{-}, 379\left[\mathrm{M}+\mathrm{Na}-\mathrm{H}_{2} \mathrm{O}\right]^{+}, 217\left[\mathrm{M}+\mathrm{Na}-\mathrm{H}_{2} \mathrm{O}-\mathrm{Glc}^{+}, 210\left[\mathrm{M}-\mathrm{H}-\mathrm{Glc}^{-}, 172[\mathrm{M}+\right.\right.$ $\left.\mathrm{Na}-\mathrm{H}_{2} \mathrm{O}-\mathrm{Glc}-\mathrm{CO}_{2}\right]^{+}, 166\left[\mathrm{M}-\mathrm{H}-\mathrm{Glc}-\mathrm{CO}_{2}\right]^{-}, 148\left[\mathrm{M}-\mathrm{H}-\mathrm{Glc}-\mathrm{CO}_{2}-\mathrm{H}_{2} \mathrm{O}\right]^{-}, 122$ $\left[\mathrm{M}-\mathrm{H}-\mathrm{Glc}-\mathrm{C}_{3} \mathrm{H}_{4} \mathrm{O}_{3}\right]^{-}$. It is a iridoid already identified in fruit of Gardenia jasminoides Ellis (Rubiaceae) [19,22,31,32] and Wendlandia formosana Cowan leaves [33].

Tarenoside $\left(\mathrm{MM}=358, \mathrm{C}_{16} \mathrm{H}_{25} \mathrm{O}_{9}\right.$, Table 1): showed signals at $357[\mathrm{M}-\mathrm{H}]^{-} ; 393[\mathrm{M}+\mathrm{Cl}]^{-}, 194[\mathrm{M}-$ $\mathrm{H}-\mathrm{Glc}^{-}$. This compound is an iridoid already identified in Wendlandia formosana Cowan leaves and Genipa americana $[2,33]$.

Loganic acid (MM = 376, $\mathrm{C}_{16} \mathrm{H}_{24} \mathrm{O}_{10}$, Table 1): showed signals at $375[\mathrm{M}-\mathrm{H}]^{-} ; 213$ [M - H - Glc]; 169, 125. It had already been described in Anthocephalus chinensis (Rubiaceae) and Ophiorrhiza liukiuensis (Rubiaceae) [2]. Loganic acid is described for the first time to Genipa genus.

Chlorogenic acid $\left(\mathrm{MM}=354, \mathrm{C}_{16} \mathrm{H}_{18} \mathrm{O}_{9}\right.$, Table 1), showed signals at $355[\mathrm{M}+\mathrm{H}]^{+}, 353[\mathrm{M}-\mathrm{H}]^{-}$, 162 [caffeic acid], 190 [quinic acid]. It had already been isolated in fruit of Gardenia jasminoides Ellis (Rubiaceae) [31,34], but the first time is described in the Genipa genus.

Kaempferol-3-O-hexoside-deoxyhexoside-7-O-deoxyhexoside (GAF 4, $\mathrm{MM}=740, \mathrm{C}_{33} \mathrm{H}_{39} \mathrm{O}_{20}$, Table 1), showed signals at $741[\mathrm{M}+\mathrm{H}]^{+}, 739[\mathrm{M}-\mathrm{H}]^{-}, 595,593[\mathrm{M}-\mathrm{Glc}]^{-}$, by scission of the robinose glycan residue to yield the radical anion $433[\mathrm{M}-\mathrm{Glc}-\mathrm{Glc}]^{-}$, 287; radical anion fragments by loss of the rhamnose glycan residue to yield the radical anion 285 [M - Glc - Glc - Glc] ${ }^{-}$[25].

Isorhamnetin-3-O-hexoside-deoxyhexoside-7-O-deoxyhexoside (GAF 5, MM = 770, $\mathrm{C}_{34} \mathrm{H}_{42} \mathrm{O}_{21}$, Table 1), showed signals at $769[\mathrm{M}-\mathrm{H}]^{-} ; 771[\mathrm{M}-\mathrm{H}]^{+} ; 625[\mathrm{M}-\mathrm{Rha}+\mathrm{H}]^{+} ; 463 ; 623[\mathrm{M}-\mathrm{Rha}-\mathrm{H}]^{-} ; 317$; 315 [M - Rha - Rha - Glc-H] ${ }^{-}$[35].

Quercetin-3-O-hexoside-deoxyhexoside (GAF 1, $\mathrm{MM}=610, \mathrm{C}_{27} \mathrm{H}_{30} \mathrm{O}_{16}$, Table 1), showed signals at $m / z$ $611[\mathrm{M}+\mathrm{H}]^{+}, 609[\mathrm{M}-\mathrm{H}]^{-}, 465$, corresponding to the loss of rhamnose unit, $301\left[\mathrm{M}-\mathrm{Glc}-\mathrm{Glc}^{-}\right.$, corresponding to the loss of the rhamnosylgalactose unit, and 303. It is suggested that the sugar is of galactose isomerism, since this form was isolated, and plants usually produces a conformation of sugar glucose or galactose, not being the rutin.

1,3-Di-O-caffeoylquinic acid (cynarine, $\mathrm{MM}=516, \mathrm{C}_{25} \mathrm{H}_{24} \mathrm{O}_{12}$, Table 1): showed signals at $515[\mathrm{M}-\mathrm{H}]^{-}$, 353 [chlorogenic acid]; 190 [acid quinic]; 178. This compound is a chlorogenic acid derivatives. It had already been isolated in Cynara cardunculus leaves [36].

Teneoside $A\left(\mathrm{MM}=518, \mathrm{C}_{22} \mathrm{H}_{30} \mathrm{O}_{14}\right.$, Table 1): showed signals at $519[\mathrm{M}+\mathrm{H}]^{+}, 373[\mathrm{M}-\mathrm{Ram}+\mathrm{H}]^{+}, 211$ $[\mathrm{M}-\mathrm{Ram}-\mathrm{Glc}+\mathrm{H}]^{+}, 517[\mathrm{M}-\mathrm{H}]^{-}, 371[\mathrm{M}-\mathrm{Ram}-\mathrm{H}]^{-}$. This is an iridoid glucoside, and it has already been isolated in Hedyotis tenelliflora Blume (Rubiaceae) [2], but is also described for the first time in the Genipa genus.

Kaempferol-3-O-hexoside-deoxyhexoside ( $\mathrm{GAF} 2, \mathrm{MM}=594, \mathrm{C}_{27} \mathrm{H}_{30} \mathrm{O}_{16}$, Table 1) showed signals at 595 $[\mathrm{M}+\mathrm{H}]^{+}, 593[\mathrm{M}-\mathrm{H}]^{-}$, and $449[\mathrm{M}-\mathrm{Glc}]^{-}$due to the loss of rhamnose, and $288[\mathrm{M}-\mathrm{Glc}]^{-}$due to the loss of the rhamnosylgalactose unit. Likewise, it is suggested that the sugar is galactose, not being, therefore the Kaempferol-3-O-rutinosideo.

Isorhamnetin-3-O-hexoside-deoxyhexoside ( $\mathrm{GAF} 3, \mathrm{MM}=624, \mathrm{C}_{28} \mathrm{H}_{33} \mathrm{O}_{16}$, Table 1) showed signals at 625 $[\mathrm{M}+\mathrm{H}]^{+}, 623[\mathrm{M}-\mathrm{H}]^{-}, 479,315$, and 317 [37]. 
The present study contributed to describe a new phytochemical approach for Genipa americana, considering that until this moment most of the studies reported only the presence of iridoids $[2,4,24]$.

\subsection{Isolation of Major Compounds}

In order to verify the positions of the sugars of the $O$-glycosides present in the flavonoids identified by HPLC-MS/MS (Section 2.3), the major constituents of the EtOAc and $n$-BuOH fractions were isolated and identified.

Based on the chromatographic profile observed by TLC and UHPLC analysis, EtOAc fraction $(3.27 \mathrm{~g})$ was chosen initially to be fractionated by vacuum liquid chromatography (VLC) and then fractions 8 and 9 were gathered and chromatographed by classical column using silica gel $(0.063-0.200 \mathrm{~mm})$ and Sephadex LH-20 gel, which yielded subfractions 134. Then subfraction (61-111) was gathered and isolated by preparative HPLC and three well-separated peaks were obtained in the chromatogram. Thereby, the following flavonoids were purified: quercetin-3-O-robinoside (GAF 1, $4.2 \mathrm{mg}$ ), kaempferol-3-O-robinoside (GAF 2, $4.0 \mathrm{mg}$ ) and isorhamnetin-3-O-robinoside (GAF 3, $6.0 \mathrm{mg}$ ).

Since the $n-\mathrm{BuOH}$ fraction $(5.0 \mathrm{~g})$ also had a flavonoid rich phytochemical profile (Figure 1 ) and higher yield, it was submitted to fractionation by VLC with silica gel, which yielded 11 subfractions. The fraction $4(1.07 \mathrm{~g})$ was isolated by classical column chromatography, and its sub-fraction 4-E of chromatography $(1.0 \mathrm{~g})$ was dissolved in methanol and chilled to $2{ }^{\circ} \mathrm{C}$, for five days, which promoted the crystallization of a part of the sample, allowed its separation. These fractions were named fraction 4-E.1 (crystallized) and fraction 4-E.2 (not crystallized). The fraction 4-E.1 was submitted to preparative HPLC and two flavonoids were further separated: kaempferol-3-O-robinoside-7-O-rhamnoside (robinin) (GAF 4, 10.0 mg) and isorhamnetin-3-O-robinoside-7-O-rhamnoside (GAF 5, $9.0 \mathrm{mg}$ ). Under the above conditions, a satisfactory separation of targeted compounds was obtained.

The chemical structures of these five compounds were elucidated by MS and $1 \mathrm{D}$ and $2 \mathrm{D}{ }^{1} \mathrm{H}$ - and ${ }^{13} \mathrm{C}-\mathrm{NMR}$ spectroscopic analyses. Comparison with literature data allowed to confirm the structures of the compounds GAF 1, 2, 3, 4, and 5 as flavonoids (Figure 2).

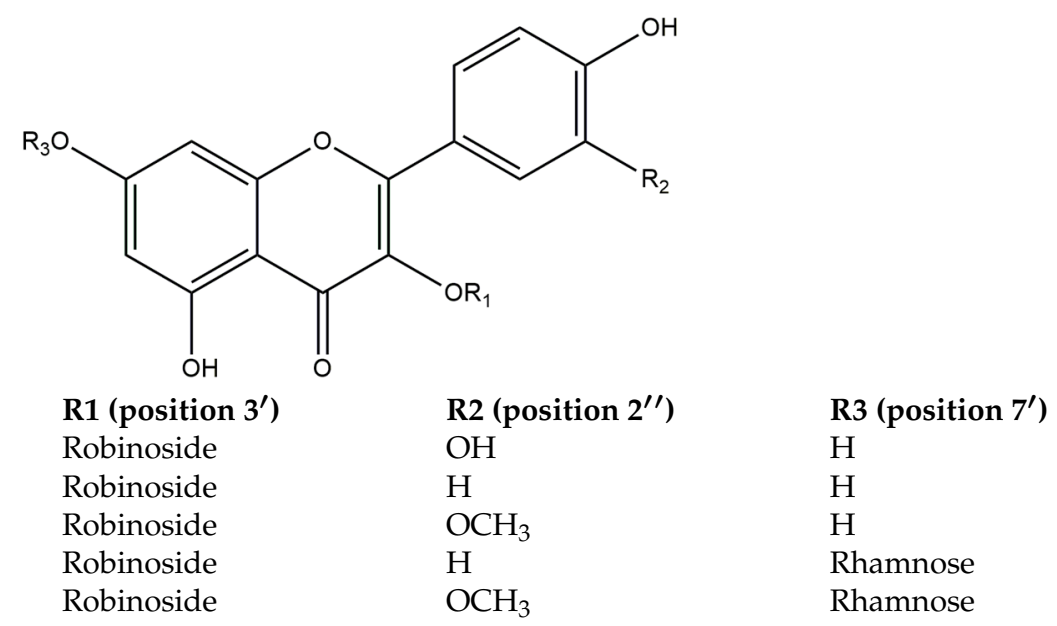

$\begin{array}{llll}\text { GAF } 1 & \text { Robinoside } & \mathrm{OH} & \mathrm{H} \\ \text { GAF 2 } & \text { Robinoside } & \mathrm{H} & \mathrm{H} \\ \text { GAF 3 } & \text { Robinoside } & \mathrm{OCH}_{3} & \mathrm{H} \\ \text { GAF 4 } & \text { Robinoside } & \mathrm{H} & \text { Rhamnose } \\ \text { GAF } 5 & \text { Robinoside } & \mathrm{OCH}_{3} & \text { Rhamnose }\end{array}$

Figure 2. Chemical structures of flavonoids GAF 1-5 isolated from the leaves of Genipa americana.

In the study, a simple and effective procedure allowed the isolation of flavonoids from the leaves of G. americana. Previously only two papers described the detection of flavonoids in fruits $[13,28]$ and one study published by our group identified these compounds in leaves [24] from G. americana. It is important to mention that this is a new phytochemical approach concerning the leaves of this species. Flavonoids are products of secondary metabolism in plants and are of interest to the pharmaceutical and food industries because of their reported wide range of biological effects [26], and they have long been associated with good health benefits, which could be attributed to their antioxidant capabilities [26]. 


\subsection{Identification of the Isolated Compounds}

To confirm the positions of the sugars of the O-glycosylated flavonoids identified by HPLCMS/MS, major compounds from EtOAc and $n$-BUOH fractions were isolated, obtaining five flavonoids. Their structures were identified by comparison of their spectroscopic data reported, including ESI-MS and NMR data.

Quercetin 3-O-robinoside [38] (GAF 1, Figure 2): $\lambda_{\max } 255,366 \mathrm{~nm}$. It was assigned with a molecular formula $\mathrm{C}_{27} \mathrm{H}_{30} \mathrm{O}_{16}$ of ESI-MS, $m / z 609.1403[\mathrm{M}-\mathrm{H}]^{-} .{ }^{1} \mathrm{H}-\mathrm{NMR}\left(400 \mathrm{MHz}, \mathrm{DMSO}-d_{6}\right) \delta: 7.66(1 \mathrm{H}$, $\left.\mathrm{dd}, J=8.5,2.2 \mathrm{~Hz}, \mathrm{H}-6^{\prime}\right), 7.53\left(1 \mathrm{H}, \mathrm{d}, J=2.2 \mathrm{~Hz}, \mathrm{H}-2^{\prime}\right), 6.82\left(1 \mathrm{H}, \mathrm{d}, J=8.5 \mathrm{~Hz}, \mathrm{H}-5^{\prime}\right), 6.39(1 \mathrm{H}, \mathrm{s}, \mathrm{H}-8)$, $6.19(1 \mathrm{H}, \mathrm{s}, \mathrm{H}-6), 5.32\left(1 \mathrm{H}, \mathrm{d}, J=7.7 \mathrm{~Hz}\right.$, galactosyl $\left.\mathrm{H}-\mathrm{1}^{\prime \prime}\right), 4.42\left(1 \mathrm{H}, \mathrm{d}, J=1.3 \mathrm{~Hz}\right.$, rhamnosyl H- $\left.{ }^{\prime \prime \prime}\right)$, $3.10\left(2 \mathrm{H}, \mathrm{t}, J=9.5 \mathrm{~Hz}, \mathrm{H}-4^{\prime \prime \prime}\right), 1.07\left(3 \mathrm{H}, \mathrm{d}, J=6.2 \mathrm{~Hz}, \mathrm{H}-6{ }^{\prime \prime \prime}\right.$, rhamnosyl-Me). ${ }^{13} \mathrm{C}-\mathrm{NMR}(100 \mathrm{MHz}$, DMSO-d 6 ) $\delta: 177.35$ (C-4), 164.25 (C-7), 161,20 (C-5), 156.38 (C-9), 156.26 (C-2), 148.56 (C-4'), 144.85 $\left(\mathrm{C}-3^{\prime}\right), 133.47(\mathrm{C}-3), 121.93\left(\mathrm{C}-6^{\prime}\right), 121.03\left(\mathrm{C}-1^{\prime}\right), 115.96\left(\mathrm{C}-2^{\prime}\right), 115.19\left(\mathrm{C}-5^{\prime}\right), 103.77(\mathrm{C}-10), 102.07\left(\mathrm{C}-1^{\prime \prime}\right)$, $99.99\left(\mathrm{C}-1^{\prime \prime \prime}\right), 98.80(\mathrm{C}-6), 93.58(\mathrm{C}-8), 73.54\left(\mathrm{C}-5^{\prime \prime}\right), 73.06\left(\mathrm{C}-3^{\prime \prime}\right), 71.92\left(\mathrm{C}-4^{\prime \prime \prime}\right), 71.10\left(\mathrm{C}-2^{\prime \prime}\right), 70.62\left(\mathrm{C}-3^{\prime \prime \prime}\right)$, $70.43\left(\mathrm{C}-2^{\prime \prime \prime}\right), 68.28\left(\mathrm{C}-5^{\prime \prime \prime}\right), 68.04\left(\mathrm{C}-4^{\prime \prime}\right), 65.09\left(\mathrm{C}-6^{\prime \prime}\right), 17.92\left(\mathrm{C}-6^{\prime \prime \prime}\right)$. The ${ }^{13} \mathrm{C}-\mathrm{NMR}$ spectrum showed the sugars to be in the $\beta$-D-galactopyranose and $\alpha$-L-rhamnopyranose forms.

The compounds GAF 1 has already been identified in: Alternanthera brasiliana (L.) Kuntze (Amaranthaceae) [37]; Strychnos variabilis (Loganiaceae) leaves; Robinia pseudacacia (Fabaceae) fruits; Lespedeza hedysaroides (Fabaceae) epigeal part; Costus sanguineus (Costaceae)leaves; Crataegus pinnatifida (Rosaceae) flowers; Brickellia chlorolepis (Asteraceae) leaves [38]; Lysimuchia vulgaris (Primulaceae) [39]; and Aspalathus linearis (Fabaceae) [40]. No reports were found for the Rubiaceae family.

Kaempferol-3-O-robinoside [38,41] (GAF 2, Figure 2): $\lambda_{\max } 265,355 \mathrm{~nm}$. It was assigned with a molecular formula $\mathrm{C}_{27} \mathrm{H}_{30} \mathrm{O}_{16}$ by ESI-MS, $m / z 593.1508[\mathrm{M}-\mathrm{H}]^{-} .{ }^{1} \mathrm{H}-\mathrm{NMR}\left(400 \mathrm{MHz}, \mathrm{DMSO}-d_{6}\right) \delta: 8.05(2 \mathrm{H}$, d, $J=8.8 \mathrm{~Hz}, \mathrm{H}-2^{\prime}$, H-6' $), 6.86\left(2 \mathrm{H}, \mathrm{d}, J=8.8 \mathrm{~Hz}, \mathrm{H}-3^{\prime}, \mathrm{H}-5^{\prime}\right), 6.41(1 \mathrm{H}, \mathrm{s}, \mathrm{H}-8), 6.19(1 \mathrm{H}, \mathrm{s}, \mathrm{H}-6), 5.31$ $(1 \mathrm{H}, \mathrm{d}, J=7.7 \mathrm{~Hz}$, galactosyl H-1' $), 4.40\left(1 \mathrm{H}, \mathrm{s}\right.$, rhamnosyl H-1 $\left.{ }^{\prime \prime \prime}\right), 3.09\left(1 \mathrm{H}, \mathrm{t}, J=9.5 \mathrm{~Hz}, \mathrm{H}-4^{\prime \prime \prime}\right), 1.06$ $\left(2 \mathrm{H}, \mathrm{d}, J=6.1 \mathrm{~Hz}, \mathrm{H}-6^{\prime \prime \prime}\right) .{ }^{13} \mathrm{C}-\mathrm{NMR}\left(100 \mathrm{MHz}, \mathrm{DMSO}-d_{6}\right) \delta: 177.39(\mathrm{C}-4), 164.58(\mathrm{C}-7), 161.18(\mathrm{C}-5)$,

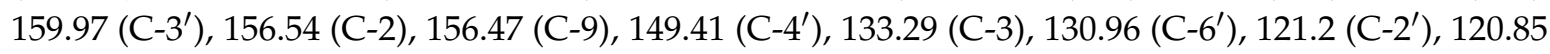
$\left(\mathrm{C}-1^{\prime}\right), 115.07\left(\mathrm{C}-5^{\prime}\right), 103.79(\mathrm{C}-10), 100.06\left(\mathrm{C}-1^{\prime \prime \prime}\right), 102.07\left(\mathrm{C}-1^{\prime \prime}\right), 98.85(\mathrm{C}-6), 93.79(\mathrm{C}-8), 73.52\left(\mathrm{C}-5^{\prime \prime}\right)$, $72.97\left(\mathrm{C}-3^{\prime \prime}\right), 71.90\left(\mathrm{C}-4^{\prime \prime \prime}\right), 71.10\left(\mathrm{C}-2^{\prime \prime}\right), 70.61\left(\mathrm{C}-2^{\prime \prime \prime}\right), 70.42\left(\mathrm{C}-3^{\prime \prime \prime}\right), 68.29\left(\mathrm{C}-5^{\prime \prime \prime}\right), 68.00\left(\mathrm{C}-4^{\prime \prime}\right), 65.30$ $\left(\mathrm{C}-6^{\prime \prime}\right), 17.93\left(\mathrm{C}-6^{\prime \prime \prime}\right)$.

The compounds GAF 2 has already been identified in: Strychnos variabilis (Loganiaceae) leaves; Atropa beladona (Solanaceae) leaves [38]; Blackstonia perfoliata (L.) (Gentianaceae) aerial parts [42]; Gynura formosana Kitam. (Asteraceae) [43]; Astragalus tana Sosn. (Fabaceae) [37]; Caragana chamlagu Lam. (Fabaceae) [37]; Alternanthera brasiliana (L.) Kuntze (Amaranthaceae) [37]; and Rumex chalepensis Mill (Polygonaceae) [41]. No reports were found for the Genipa genus.

Isorhamnetin-3-O-robinoside [37] (GAF 3, Figure 2): $\lambda_{\max } 255,370 \mathrm{~nm}$. It was assigned with a molecular formula $\mathrm{C}_{28} \mathrm{H}_{33} \mathrm{O}_{16}$ by ESI-MS, $m / z 623.1596[\mathrm{M}-\mathrm{H}]^{-} .{ }^{1} \mathrm{H}-\mathrm{NMR}\left(400 \mathrm{MHz}, \mathrm{DMSO}-d_{6}\right) \delta: 8.00(1 \mathrm{H}$, $\left.\mathrm{d}, J=2.3 \mathrm{~Hz}, \mathrm{H}-2^{\prime}\right), 7.51\left(1 \mathrm{H}, \mathrm{dd}, J=8.4,2.1 \mathrm{~Hz} ; \mathrm{H}-6^{\prime}\right), 6.90\left(1 \mathrm{H}, \mathrm{d}, J=8.3 \mathrm{~Hz}, \mathrm{H}-5^{\prime}\right), 6.42(1 \mathrm{H}, \mathrm{s}, \mathrm{H}-8)$, $6.19(1 \mathrm{H}, \mathrm{d}, J=1.8 \mathrm{~Hz}, \mathrm{H}-6), 5.45\left(1 \mathrm{H}, \mathrm{d}, J=7.7 \mathrm{~Hz} ; \mathrm{H}-1^{\prime \prime}\right), 4.42\left(1 \mathrm{H}, \mathrm{d}, J=1.7 \mathrm{~Hz} ; \mathrm{H}-1^{\prime \prime \prime}\right), 3.85(3 \mathrm{H}$, s, H-3'-O-CH 3$), 3.08\left(2 \mathrm{H}, \mathrm{t}, J=9.4 \mathrm{~Hz}, \mathrm{H}-4^{\prime \prime \prime}\right), 1.05\left(3 \mathrm{H}, \mathrm{d}, J=6.1 \mathrm{~Hz}, \mathrm{H}-6^{\prime \prime \prime}\right) .{ }^{13} \mathrm{C}-\mathrm{NMR}(100 \mathrm{MHz}$, DMSO-d 6 ) $\delta: 177.29$ (C-4), 164.65 (C-7), 161.19 (C-5), 156.46 (C-9), 156.33 (C-2), 149.45 (C-4'), 146.99 $\left(\mathrm{C}-3^{\prime}\right), 133.08(\mathrm{C}-3), 121.96\left(\mathrm{C}-6^{\prime}\right), 121.05\left(\mathrm{C}-1^{\prime}\right), 115.16\left(\mathrm{C}-5^{\prime}\right), 113.44\left(\mathrm{C}-2^{\prime}\right), 103.84(\mathrm{C}-10), 101.85\left(\mathrm{C}-1^{\prime \prime}\right)$, $100.06\left(\mathrm{C}-1^{\prime \prime \prime}\right), 98.92(\mathrm{C}-6), 93.82(\mathrm{C}-8), 73.56\left(\mathrm{C}-5^{\prime \prime}\right), 72.94\left(\mathrm{C}-3^{\prime \prime}\right), 71.14\left(\mathrm{C}-2^{\prime \prime}\right), 70.6\left(\mathrm{C}-4^{\prime \prime \prime}\right), 70.4\left(\mathrm{C}-2^{\prime \prime \prime}\right)$, $68.3\left(\mathrm{C}-5^{\prime \prime \prime}\right), 67.98\left(\mathrm{C}-4^{\prime \prime}\right), 65.18\left(\mathrm{C}-6^{\prime \prime}\right), 55.94\left(\mathrm{C}-3^{\prime}-\mathrm{O}-\mathrm{CH}_{3}\right), 17.90\left(\mathrm{C}-6^{\prime \prime \prime}\right)$.

The compounds GAF 3 is a diglycoside that has already been identified in: Gomphrena martiana Moquin (Amaranthaceae) [39]; Blackstonia perfoliata (L.) (Gentianaceae) aerial parts [42]; NitrariaRetusa (Nitrarioideae) leaves [40]; and Calotropis procera R. Br. (Asclepiadaceae) leaves [44]. No reports were found for the Rubiaceae family. 
Kaempferol-3-O-robinoside-7-O-rhamnoside (robinin) [45] (GAF 4, Figure 2): $\lambda_{\max } 266,346 \mathrm{~nm}$. It was assigned with a molecular formula $\mathrm{C}_{33} \mathrm{H}_{39} \mathrm{O}_{20}$ by ESI-MS, $m / z$ 739.2062. $[\mathrm{M}-\mathrm{H}]^{-}$. ${ }^{1} \mathrm{H}-\mathrm{NMR}$ $\left(400 \mathrm{MHz}, \mathrm{DMSO}-\mathrm{d}_{6}\right) \delta: 8.10\left(2 \mathrm{H}, \mathrm{d}, J=9.0 \mathrm{~Hz}, \mathrm{H}-2^{\prime}, 6^{\prime}\right), 6.88\left(2 \mathrm{H}, \mathrm{d}, J=9.0 \mathrm{~Hz}, \mathrm{H}-3^{\prime}, 5^{\prime}\right), 6.81(1 \mathrm{H}, \mathrm{d}, J$ $=2.1 \mathrm{~Hz}, \mathrm{H}-8), 6.46(1 \mathrm{H}, \mathrm{d}, J=2.1 \mathrm{~Hz}, \mathrm{H}-6), 5.56\left(1 \mathrm{H}, \mathrm{d}, J=1.4 \mathrm{~Hz}, \mathrm{H}-1^{\prime}\right), 5.36\left(1 \mathrm{H}, \mathrm{d}, J=7.6 \mathrm{~Hz}, \mathrm{H}-1^{\prime \prime}\right)$, $4.40\left(1 \mathrm{H}, \mathrm{d}, J=1.7 \mathrm{~Hz}, \mathrm{H}-1^{\prime \prime \prime}\right), 3.09\left(1 \mathrm{H}, \mathrm{dd}, J=12 \mathrm{~Hz}, 6.5, \mathrm{H}-4^{\prime \prime \prime}\right), 1.12\left(2 \mathrm{H}, \mathrm{d}, J=6.1 \mathrm{~Hz}, \mathrm{H}-6^{\prime \prime \prime \prime}\right), 1.05$ $\left(2 \mathrm{H}, \mathrm{d}, J=6.2 \mathrm{~Hz}, \mathrm{H}-6^{\prime \prime \prime}\right) .{ }^{13} \mathrm{C}-\mathrm{NMR}\left(100 \mathrm{MHz}, \mathrm{DMSO}-d_{6}\right) \delta: 177.66(\mathrm{C}-4), 161.64(\mathrm{C}-5), 160.19\left(\mathrm{C}-4^{\prime}\right)$, 157.08 (C-2), 156.04 (C-9), 133.57 (C-3), $131.11\left(\mathrm{C}-6^{\prime}\right), 120.71\left(\mathrm{C}-1^{\prime}\right), 115.14\left(\mathrm{C}-5^{\prime}\right), 105.60$ (C-10), 103.29 $\left(\mathrm{C}-1^{\prime \prime}\right), 100.03\left(\mathrm{C}-1^{\prime \prime \prime}\right), 99.73(\mathrm{C}-6), 98.39\left(\mathrm{C}-1^{\prime \prime \prime \prime}\right), 94.88(\mathrm{C}-8), 73.61\left(\mathrm{C}-5^{\prime \prime}\right), 72.96\left(\mathrm{C}-3^{\prime \prime}\right), 71.92\left(\mathrm{C}-4^{\prime \prime \prime}\right)$, $71.62\left(\mathrm{C}-4^{\prime \prime \prime}\right), 71.10\left(\mathrm{C}-2^{\prime \prime}, 2^{\prime \prime \prime \prime}\right), 70.60\left(\mathrm{C}-3^{\prime \prime \prime}\right), 70.43\left(\mathrm{C}-2^{\prime \prime \prime}\right), 70.27\left(\mathrm{C}-3^{\prime \prime \prime \prime}\right), 69.85\left(\mathrm{C}-5^{\prime \prime \prime \prime}\right), 68.28\left(\mathrm{C}-5^{\prime \prime \prime}\right)$, $68.00\left(\mathrm{C}-4^{\prime \prime}\right), 17.93\left(\mathrm{C}-6^{\prime \prime \prime}\right)$.

The compounds GAF 4 is a flavone triglycoside of the well-studied has already been identified in: Alternanthera brasiliana (Amaranthaceae) leaves [37]; Strychnos variabilis (Loganiaceae) leaves; Atropa beladona (Solanaceae) leaves [38]; Astragalus falcatus Lam. (Leguminosae) flowers [45]; Robinia pseudoacacia (Leguminosae) leaves [46]; and Melilotus elegans Salzm. ex Ser. (Leguminosae) leaves [47]. No reports were found for the Rubiaceae family.

Isorhamnetin-3-O-robinoside-7-O-rhamnoside [35] (GAF 5, Figure 2): $\lambda_{\max } 255,354 \mathrm{~nm}$. It was assigned with a molecular formula $\mathrm{C}_{34} \mathrm{H}_{42} \mathrm{O}_{21}$ by ESI-MS, $m / z 769.2220[\mathrm{M}-\mathrm{H}]^{-}$. ${ }^{1} \mathrm{H}-\mathrm{NMR}(400 \mathrm{MHz}$, DMSO-d 6 ) $\delta: 8.02\left(1 \mathrm{H}, \mathrm{d}, J=2.1 \mathrm{~Hz}, \mathrm{H}-2^{\prime}\right), 7.60-7.57\left(1 \mathrm{H}, \mathrm{m}, \mathrm{H}-6^{\prime}\right), 6.93-6.90\left(1 \mathrm{H}, \mathrm{m}, \mathrm{H}-5^{\prime}\right), 6.46(1 \mathrm{H}, \mathrm{d}$, $J=2.2 \mathrm{~Hz}, \mathrm{H}-8), 5.57\left(1 \mathrm{H}, \mathrm{d}, J=2.1 \mathrm{~Hz}, \mathrm{H}-1^{\prime \prime \prime \prime}\right), 5.48\left(1 \mathrm{H}, \mathrm{d}, J=7.7 \mathrm{~Hz}, \mathrm{H}-1^{\prime \prime}\right), 3.87\left(3 \mathrm{H}, \mathrm{s}, \mathrm{H}-3^{\prime}-\mathrm{O}-\mathrm{CH}_{3}\right)$, 3.12-3.05 (1H, m, H-4 $\left.{ }^{\prime \prime \prime}\right), 1.13\left(3 \mathrm{H}, \mathrm{d}, J=6.1 \mathrm{~Hz}, \mathrm{H}-6^{\prime \prime \prime \prime}\right), 1.06\left(3 \mathrm{H}, \mathrm{d}, J=6.1 \mathrm{~Hz}, \mathrm{H}-6^{\prime \prime \prime}\right) .{ }^{13} \mathrm{C}-\mathrm{NMR}$ $\left(100 \mathrm{MHz}, \mathrm{DMSO}-d_{6}\right) \delta: 178.01$ (C-4), 162.08 (C-7), 161.34 (C-5), 157.35 (C-9), $156.46(\mathrm{C}-2), 150.12\left(\mathrm{C}-4^{\prime}\right)$, $147.50\left(\mathrm{C}-3^{\prime}\right), 133.84(\mathrm{C}-3), 122.69\left(\mathrm{C}-6^{\prime}\right), 121.33\left(\mathrm{C}-1^{\prime}\right), 115.64\left(\mathrm{C}-5^{\prime}\right), 113.87\left(\mathrm{C}-2^{\prime}\right), 106.08(\mathrm{C}-10), 102.16$ $\left(\mathrm{C}-1^{\prime \prime}\right), 98.81(\mathrm{C}-6), 95.18(\mathrm{C}-8), 73.38\left(\mathrm{C}-5^{\prime \prime}\right), 72.34\left(\mathrm{C}-4^{\prime \prime \prime}\right), 71.58\left(\mathrm{C}-4^{\prime \prime \prime \prime}\right), 71.05\left(\mathrm{C}-2^{\prime \prime}\right), 70.73\left(\mathrm{C}-3^{\prime \prime \prime \prime}\right)$, $70.30\left(\mathrm{C}-3^{\prime \prime \prime}\right), 56.40\left(\mathrm{C}-3^{\prime}-\mathrm{O}-\mathrm{CH}_{3}\right), 18.38\left(\mathrm{C}-6^{\prime \prime \prime \prime}\right), 18.34\left(\mathrm{C}-6^{\prime \prime \prime}\right)$.

The compounds GAF 5 is a triglycoside that has already been identified in Rhazya stricta Decaisne (Apocynaceae) leaves [35]; and Blackstonia perfoliata (L.) (Gentianaceae) aerial parts [42].

Only after the isolation was it possible to analyze the compounds by ${ }^{1} \mathrm{H}-$ and ${ }^{13} \mathrm{C}-\mathrm{NMR}$ and to differentiate the types of sugar present, being possible to identify the final portion as galactose. In addition, it can be confirmed that the sugars are present in positions-3 (GAF 1-5) and-7 (GAF 4-5).

Compound GAF 1 exhibited in vitro inhibitory activities against leukemia K562 cells in different extents [47], and cytotoxic activity against HepG-2cells. The hydroxylation pattern of the $C$-rings of the flavonoid compounds like quercetin aglycone, play an essential role in their cytotoxic activities, especially the inhibition of protein kinase antiproliferation activity [48].

Kaempferol-3-O-robinoside (GAF 2) significantly inhibited the human lymphocyte proliferation in vitro, to a greater extent (IC50 $\cong 25 \mu \mathrm{g} \mathrm{mL}{ }^{-1}$ ) and were twice more active than crude extract of Alternanthera brasiliana [37]. The compound GAF 2 showed radical scavenging activities when evaluated using the DPPH method. The IC50 values of the DPPH radical were $286.7 \mathrm{mM} \mathrm{[43].}$

The isorhamnetin 3-O-robinoside (GAF 3) showed a protective effect against lipid peroxidation induced by $\mathrm{H}_{2} \mathrm{O}_{2}$ and antigenotoxic potential on human chronic myelogenous leukemia cell line K562 [49].

Flavonoids are considered as a class of natural products of high pharmacological potency but, unfortunately, many of them have a low solubility in water. We have also isolated one flavonol glycosides very soluble in water: robinin (GAF 4). Similar compound have been previously isolated from leaves of Atropa belladonna [38] but your structure have not been fully elucidated. Robinin (GAF 4) displayed a marked activity, inhibiting edema (38.8\%) at a concentration of $0.0027 \mathrm{mmol} / \mathrm{kg}$ of body weight, four hours after injection of carrageenin [47]. GAF 4 was also able to inhibit lymphocyte proliferation to a greater extent (IC50 $\cong 25 \mu \mathrm{g} \mathrm{mL}^{-1}$ ) and were twice more active than crude extract of Alternanthera brasiliana [37].

No reports about pharmacological activities were found for GAF 5. 
This work described the first time the isolation and identification of flavonoids in leaves at G. americana. This is an important finding for subsequent studies aimed at the standardization of leaf extracts.

\section{Materials and Methods}

\subsection{Plant Material}

The leaves of Genipa americana L., Rubiaceae, were collected in Natal City, Rio Grande do Norte State, Brazil, at coordinates lat: -6.1278 long: -35.1115 WGS22, in May 2012. The plant was identified by botanist Alan de Araújo Roque (UFRN) and a voucher specimen was deposited at the Herbarium of the Federal University of Rio Grande do Norte (UFRN), Brazil, with an identification number 12251.

The collection of the plant material was conducted under authorization of Brazilian Authorization and Biodiversity Information System (SISBIO) (process number 35017) and National System for the Management of Genetic Heritage and Associated Traditional Knowledge (SISGEN) (process number A618873).

\subsection{Extraction and TLC Characterization}

The leaves of G. americana was evaporated at $40{ }^{\circ} \mathrm{C}$ in a circulating air oven and powdered leaves $(600 \mathrm{~g})$ were extracted by maceration with ethanol:water $(70: 30, v / v)$ for five days (plant:solvent, 1.5:10, $w / v ; 4 \mathrm{~L} ;$ at room temperature). Then, the organic solvent was evaporated under reduced pressure in rotary evaporator (temperature below $45^{\circ} \mathrm{C}$ ) and water residue was freeze-dried, obtaining the hydroethanolic extract (HE).

The HE was submitted to a liquid-liquid extraction with organic solvents in order of increasing polarity: petroleum ether $(\mathrm{PE})(3 \times 200 \mathrm{~mL})$, was sequentially partitioned with chloroform $(3 \times 200 \mathrm{~mL})$, ethyl acetate $(3 \times 200 \mathrm{~mL})$ and $n$-butanol $(3 \times 200 \mathrm{~mL})$. The fractions were evaporated under reduced pressure (temperature below $45^{\circ} \mathrm{C}$ ) and respectively afforded the PE $(0.70 \mathrm{~g}), \mathrm{CHCl}_{3}(2.9589 \mathrm{~g}), \mathrm{EtOAC}$ $(3.27 \mathrm{~g})$, and $n-\mathrm{BuOH}(14.51 \mathrm{~g})$ fractions of the leaves of G. americana.

In the phytochemical screening the HE and fractions were analyzed by TLC using aluminum sheets, coated with silica gel F254 as absorbent and chromatographed with ethyl acetate:formic acid:water: methanol (10:1.6:1.5:0.6, $v / v / v / v)$ as mobile phase. The TLC was analyzed under 254 and $365 \mathrm{~nm}$ ultraviolet (UV) light and then sprayed with vanillin sulfuric acid (4\%) or natural product reagent $(0.5 \%)-\mathrm{NP}$ reagent.

\subsection{UHPLC Characterization}

The samples were analyzed by ultra-high performance liquid chromatography coupled with a diode array detector (UHPLC/HPLC-DAD), model UFLC (Shimadzu, Kyoto, Japan), containing a quaternary pump system (LC-20A 3 XR), equipped with a degasser (DGU-20A 3 ), auto-sampler (SIl-20AC $\mathrm{XR}$ ), column oven (CTO-20AC), and diode-array detectors (SPD-M20A), with Software LC Solution (Shimadzu, Kyoto, Japan) controlled system. A C 18 column Shim-pack XR-ODS $30 \times 2 \mathrm{~mm}, 2.2 \mu \mathrm{m}$ (Shimadzu, Kyoto, Japan); a temperature of $25 \pm 2{ }^{\circ} \mathrm{C}$ was used for the analysis and separation of the compounds and was achieved using a solvent system mixture of acetonitrile: acidified water (with $0.3 \%$ formic acid) as the mobile phase with a flow rate of $0.3 \mathrm{~mL} / \mathrm{min}$, and a detector was set at $254 \mathrm{~nm}$.

\subsection{HPLC-ESI-IT-MS/MS Characterization}

The hydroethanolic extracts and EtOAc and $\mathrm{BuOH}$ fractions were analyzed by HPLC-IT-MS/MS. UFLC (Shimadzu, Kyoto, Japan) containing two LC20AD solvent pumps, a SIL20AHT auto sampler, a SPD-M20A detector and a CBM20A system controller, coupled with an ion-trap mass spectrometer (AmaZon X, Bruker, Billerica, MA, USA). LC experiments were performed using a $\mathrm{C}_{18}$ column (Kromasil-250 $\mathrm{mm} \times 4.6 \mathrm{~mm} \times 5 \mu \mathrm{m}$ ) and the following gradient elution: solvent A: water and formic acid $(0.1 \%, v / v)$; solvent B: acetonitrile; injection volume of $20 \mu \mathrm{L}$, and flow rate of $0.6 \mathrm{~mL} / \mathrm{min}$. 
The ion-trap analysis parameters are as follows: capillary $4.5 \mathrm{kV}$, ESI in positive mode, final plate offset $500 \mathrm{~V}, 40$ psi nebulizer, dry gas $\left(\mathrm{N}_{2}\right)$ with flow rate of $8 \mathrm{~mL} / \mathrm{min}$ and a temperature of $300{ }^{\circ} \mathrm{C}$. CID fragmentation was achieved in auto MS/MS mode using advanced resolution mode for MS and MS/MS mode. The spectra $(m / z 50-1000)$ were recorded every two seconds.

The data obtained were interpreted with the help of the following: Metlin, MassBank and Scienfinder.

\subsection{Isolation of Major Compounds}

According to their profiles by TLC, the EtOAc and $n$ - $\mathrm{BuOH}$ fractions were gathered for subsequent isolation. Fractionation of the compounds of G. americana started with the EtOAc fraction ( $3.27 \mathrm{~g})$, which was submitted to vacuum liquid chromatography (VLC) $(10 \times 15 \mathrm{~cm})$ on a sintered funnel filled with silica gel $60(0.063-0.200 \mathrm{~mm})$ and eluted with $n$-hexane (50:10):methanol (50:50):water $(10: 100) v / v, 150 \mathrm{~mL}$. This procedure yielded in 10 fractions. In this step, the fractions that showed the presence of flavonoids in TLC analysis were chosen for isolation. Fractions 8 and 9 of VLC were gathered $(1.5 \mathrm{~g})$ and further subjected to classical column chromatography $(25 \times 3 \mathrm{~cm})$ in a Sephadex LH-20(GE Healthcare Bio-Science AB, Uppsala, Sweden), eluted with chloroform:water:methanol (9:0.1:0.9; $v / v ; 2.0 \mathrm{~mL} / \mathrm{min}$ ) affording 134 fractions. Fractions 61-111 were gathered (Fr. 61-111, $153 \mathrm{mg}$ ) and submitted to purification by HPLC (mobile phase: acetonitrile (21-23\%) using a Shimadzu Shimpack ODS $(\mathrm{H}) \mathrm{kit}, \mathrm{C}_{18}$ column $(200 \mathrm{~mm} \times 20 \mathrm{~mm}, 5 \mu \mathrm{m})$, flow rate $10 \mathrm{~mL} / \mathrm{min}$ and UV $254 \mathrm{~nm}$, $270 \mathrm{~nm}$ and $340 \mathrm{~nm}$ ) (for details, see Section 3.6). This procedure yielded compounds GAF 1 (4.2 $\mathrm{mg}$ ), GAF 2 (4.0 mg) and GAF 3 (6.0 mg).

The $n$-BuOH fraction $(5.0 \mathrm{~g})$ was submitted to VLC $(10 \times 15 \mathrm{~cm})$ on silica gel $60(0.063-0.200 \mathrm{~mm})$ and eluted with gradient-mode methanol:EtOAc $(0-100 \% ; v / v)$. This procedure resulted in 11 fractions. Fraction $4(1.07 \mathrm{~g})$ was subjected to classical column chromatography $(25 \times 3 \mathrm{~cm})$ on silica gel 60 $(0.063-0.200 \mathrm{~mm})$ and eluted with EtOAc:formic acid:water:methanol (6.5:1.4:1:1; v/v), sub-fraction 4-E showed greater intensity of phenolic compounds in analysis by TLC, being selected for isolation steps. The fraction 4-E of chromatography $(1.0 \mathrm{~g})$ was dissolved in methanol and chilled to $2{ }^{\circ} \mathrm{C}$, for five days, which promoted the crystallization of a part of the sample, which was separated and named fraction 4-E.1 (crystallized, $60 \mathrm{mg}$ ) and fraction 4-E.2 (not crystallized, $440 \mathrm{mg}$ ). The sub-fraction 4-E.2 was submitted to preparative HPLC developed with wateracetonitrile $(0-30 \mathrm{~min}, 18 \% ; 10 \mathrm{~mL} / \mathrm{min})$ (for details, see Section 3.6) using $C_{18}$ column ( $200 \mathrm{~mm} \times 20 \mathrm{~mm}, 5 \mu \mathrm{m}$ ), and UV $254 \mathrm{~nm}, 270 \mathrm{~nm}$, and $340 \mathrm{~nm}$. This procedure yielded compounds GAF $4(10.0 \mathrm{mg})$ and GAF $5(9.0 \mathrm{mg})$.

\subsection{Preparative HPLC Optimization and Analyses}

In order to obtain the isolate compounds, these fractions were subjected to preparative HPLC for further purification. The preparative HPLC separations were used with a $\mathrm{C}_{18}$ column $(200 \mathrm{~mm} \times$ $20 \mathrm{~mm}, 5 \mu \mathrm{m}$ ); and mobile phase was selected based on the polarity of the likely compounds and the analytical HPLC conditions. Several mobile phases composed of acetonitrile (B)-water(A) in various concentrations of acetonitrile $(15 \%, 18 \%, 20 \%, 21 \%, 23 \%, 24 \%, 25 \%, 30 \%)$ were tested. The results indicated that the best separation conditions were achieved using acetonitrile in a gradient mode (0-28 $\mathrm{min}, 21-23 \%)$ for the ethyl acetate fraction and isocratic mode (0-30 $\mathrm{min}, 18 \%)$ for $n$ - $\mathrm{BuOH}$ fraction and a flow rate of $10 \mathrm{~mL} / \mathrm{min}$ and a monitoring wavelength of $254 \mathrm{~nm}, 270 \mathrm{~nm}$ and $340 \mathrm{~nm}$, with LabSolutions software (Shimadzu, Kyoto, Japan). Under the above conditions, a satisfactory separation of each the targeted compounds was achieved.

\subsection{MS/MS of Isolated Compounds}

The isolated compounds were analysis by MS-MS in positive and negative modes were by mass of direct infusion the microTOF II-ESI-TOF-Bruker Daltonics (Bruker, Billerica, MA, USA), with a drying gas flow rate o $\mathrm{f} 4 \mathrm{~L} / \mathrm{min}$ at $180^{\circ} \mathrm{C}$, nebulizer gas 0.4 bar (pressure), internal calibration standard: TFA, and syringe flow: $10 \mu \mathrm{L} / \mathrm{min}$. 


\subsection{Nuclear Magnetic Resonance Spectroscopy (NMR) of Isolated Compounds}

The nuclear magnetic resonance (NMR) spectra were performed on a (Bruker, Billerica, MA, USA) $\left(400 \mathrm{MHz}\right.$ for ${ }^{1} \mathrm{H}$ and $100 \mathrm{MHz}$ for ${ }^{13} \mathrm{C}$ ) and chemical shifts are given in ppm relative to residual DMSO- $d_{6}(2.5)$, and to the central peak of the triplet related to DMSO- $d_{6}$ carbon (39.5 ppm).

\section{Conclusions}

Through characteristic fragmentation patterns of substances obtained by MS/MS data, 13 compounds were identified. The flavonoids were isolated and identified.

In contrast to literature, which describes mainly the presence of iridoids for the fruit and leaf extracts of G. americana, in this paper the leaves showed to be rich in O-glycosidic flavonoids. The isolation was carried out in few steps and allowed the identification of five flavonoids glycosides not described until this moment to the G. americana leaf extract. These flavonoids GAF 1, 3, 4, and 5 were identified for the first time in the Rubiaceae family and flavonoid GAF $\mathbf{2}$ is unknown for genus Genipa. All flavonol aglycones have sugars units, attached only at the 3-position. The similarities of the compounds of five chemical structures suggest a common biosynthetic pathway in this species. The present article was conducted to evaluate the chromatograph profiles of the leaf extract from Genipa americana, to be used in future to the quality control for this species. It can also be suggesting that flavonoids identified for this species may be associated, at least in part, to pharmacological properties of the plant.

Author Contributions: L.M.P.S. performed the experiments, analyzed the results, identified the compounds, and wrote the manuscript; J.S.F.A. performed the isolation of the chemical compounds; D.L.P. performed the characterization by UHPLC-DAD; C.F.S.A. and J.F.T. contributed the reagents/materials/analysis tools; L.S.A., E.M.d.S.S., M.A.d.S.N., N.P.L., L.d.S.F. and D.P.D. helped in the identification of the compounds; S.M.Z. conceived and designed the experiments, analyzed the data, interpreted the results, and wrote the manuscript. All the authors read and approved the final manuscript.

Funding: We thank the Conselho Nacional de Desenvolvimento Científico e Tecnológico-CNPq 478661/2010-e Coordenação de Aperfeiçoamento de Pessoal de Nível Superior -CAPES for funding this project and UFRN-Universidade Federal do Rio Grande do Norte for support.

Acknowledgments: The authors acknowledge all contributors for their valuable time and commitment to the study.

Conflicts of Interest: The authors declare no conflict of interest.

\section{References}

1. Barbosa, M.R.; Zappi, D.; Taylor, C.; Cabral, E.; Jardim, J.G.; Pereira, M.S.; Calió, M.F.; Pessoa, M.C.R.; Salas, R.; Souza, E.B.; et al. Rubiaceae em Flora do Brasil 2020 em construção.Jardim Botânico do Rio de Janeiro. Available online: http:/ / floradobrasil.jbrj.gov.br/jabot/FichaPublicaTaxonUC/FichaPublicaTaxonUC.do? id=FB14045 (accessed on 8 March 2018).

2. Martins, D.; Nunez, C.V. Secondary metabolites from rubiaceae species. Molecules 2015, 20, 13422-13495. [CrossRef] [PubMed]

3. Santos, O.A.; Couceiro, S.R.M.; Rezende, A.C.C.; Silva, M.D.S. Composition and richness of woody species in riparian forests in urban areas of Manaus, Amazonas, Brazil. Landsc. Urban Plan 2016, 150, 70-78. [CrossRef]

4. Ueda, S.; Iwahashi, Y. Production of anti-tumor-promoting iridoid glucosides in Genipa americana and its cell cultures. J. Nat. Prod. 1991, 54, 1677-1680. [CrossRef] [PubMed]

5. Zappi, D. Genipa in Lista de Espécies da Flora do Brasil. Jardim Botânico do Rio de Janeiro. Available online: http:/ / floradobrasil.jbrj.gov.br/jabot/floradobrasil/FB14045 (accessed on 22 November 2017).

6. Almeida, E.R.; Almeida, E.; Almeida, E. Plantas Medicinais Brasileiras: Conhecimentos Populares e Científicos; Hemus: São Paulo, Brasil, 1993; pp. 215-216.

7. Gomes, R.P. Fruticultura Brasileira; Nobel: São Paulo, Brasil, 1982; pp. 278-281.

8. Agra, M.F.; Silva, K.N.; Basílio, I.J.L.D.; de Freitas, P.F.; Barbosa-Filho, J.M. Survey of medicinal plants used in the region Northeast of Brazil. Rev. Bras. Farmacogn. 2008, 18, 472-508. [CrossRef] 
9. Mors, W.B.; Rizzini, C.T.; Pereira, N. Medicinal Plants of Brazil; Reference Publications, Inc.: Algonac, MI, USA, 2000.

10. Delprete, P.G.; Smith, L.B.; Klein, R.M. Rubiáceas. In Flora Ilustrada Catarinense; Reis, A., Ed.; Herbário Barbosa Rodrigues: Itajaí, Santa Catarina, Brizal, 2005.

11. Koo, H.J.; Song, Y.S.; Kim, H.J.; Lee, Y.H.; Hong, S.M.; Kim, S.J.; Kim, B.C.; Jin, C.; Lim, C.J.; Park, E.H. Antiinflammatory effects of genipin, an active principle of gardenia. Eur. J. Pharmacol. 2004, 495, 201-208. [CrossRef] [PubMed]

12. Kim, B.C.; Kim, H.G.; Lee, S.A.; Lim, S.; Park, E.H.; Kim, S.J.; Lim, C.J. Genipin-induced apoptosis in hepatoma cells is mediated by reactive oxygen species/c-Jun NH 2-terminal kinase dependent activation of mitochondrial pathway. Biochem. Pharmacol. 2005, 70, 1398-1407. [CrossRef] [PubMed]

13. Nogueira, F.A.; Nery, P.S.; Morais-Costa, F.; de Faria Oliveira, N.J.; Martins, E.R.; Duarte, E.R. Efficacy of aqueous extracts of Genipa americana L. (Rubiaceae) in inhibiting larval development and eclosion of gastrointestinal nematodes of sheep. J. Appl. Anim. Res. 2014, 42, 356-360. [CrossRef]

14. De Souza, P.M.; De Salesi, P.M.; Simeoni, L.A.; Silva, E.C.; Silveira, D.; Magalhães, P.O. Inhibitory activity of $\alpha$-amylase and $\alpha$-glucosidase by plant extracts from the Brazilian cerrado. Planta Med. 2012, 78, 393-399. [CrossRef] [PubMed]

15. Nonato, D.T.T.; Vasconcelos, S.M.M.; Mota, M.R.L.; de Barros Silva, P.G.; Cunha, A.P.; Ricardo, N.M.P.S.; Pereira, M.G.; Assreuy, A.M.S.; Chaves, E.M.C. The anticonvulsant effect of a polysaccharide-rich extract from Genipa americana leaves is mediated by GABA receptor. Biomed. Pharmacother. 2018, 101, 181-187. [CrossRef] [PubMed]

16. Ono, M.; Ishimatsu, N.; Masuoka, C.; Yoshimitsu, H.; Tsuchihashi, R.; Okawa, M.; Kinjo, J.; Ikeda, T.; Nohara, T. Three new monoterpenoids from the fruit of Genipa americana. Chem. Pharm. Bull. 2007, 55, 632-634. [CrossRef] [PubMed]

17. De Bentes, A.S.; Mercadante, A.Z. Influence of the stage of ripeness on the composition of iridoids and phenolic compounds in genipap (Genipa americana L.). J. Agric. Food Chem. 2014, 5, 62. [CrossRef] [PubMed]

18. Djerassi, C.; Gray, J.D.; Kincl, F. Isolation and characterization of genipin. J. Org. Chem. 1960, 25, $2174-2177$. [CrossRef]

19. Ono, M.; Ueno, M.; Masouka, C.; Ikeda, T.; Nohara, T. Iridoid glucosides from the fruit of Genipa americana. Chem. Pharm. Bull. 2005, 53, 1342-1344. [CrossRef] [PubMed]

20. Tallent, W.H. Two new antibiotic cyclopentanoid monoterpenes of plant origin. Tetrahedron Lett. 1964, 20, 1781-1787. [CrossRef]

21. Hsua, H.; Yang, J.; Lin, S.; Linb, C. Comparisons of geniposidic acid and geniposide on antitumor and radioprotection after sublethal irradiation. Cancer Lett. 1997, 113, 31-37. [CrossRef]

22. Guarnaccia, R.; Madyastha, K.M.; Tegtmeyer, E.; Coscia, C.J. Geniposidic acid, an iridoid glucoside from Genipa americana. Tetrahedron Lett. 1972, 50, 5125-5127. [CrossRef]

23. Hossain, C.F.; Jacob, M.R.; Clark, A.M.; Walker, L.A.; Nagle, D.G. Genipatriol, a new cycloartane triterpene from Genipa spruceana. J. Nat. Prod. 2003, 66, 398-400. [CrossRef] [PubMed]

24. Alves, J.S.F.; Medeiros, L.A.; Fernandes-Pedrosa, M.F.; Araújo, R.M.; Zucolotto, S.M. Iridoids from leaf extract of Genipa americana. Rev. Bras. Farmacogn. 2017, 27, 641-644. [CrossRef]

25. March, R.E.; Miao, X.-S.; Metcalfe, C.D. A fragmentation study of a flavone triglycoside, kaempferol-3-Orobinoside-7-O-rhamnoside. Rapid Commun. Mass Spectrom. 2004, 18, 931-934. [CrossRef] [PubMed]

26. Panche, A.N.; Diwan, A.D.; Chandra, S.R. Flavonoids: An overview. J. Nutr. Sci. 2016, 5, 1-15. [CrossRef] [PubMed]

27. Wagner, H.; Bladt, S. Plant Drug Analysis: A Thin Layer Chromatography Atlas; Springer: Berlin, Germany, 2001.

28. Omena, C.M.B.; Valentim, I.V.; Guedes, G.S.; Rabelo, L.A.; Mano, C.M.; Bechara, E.J.H.; Sawaya, A.C.H.F.; Trevisan, M.T.S.; Da Costa, J.G.; Ferreira, R.C.S.; et al. Antioxidant, anti-acetylcholinesterase and cytotoxic activities of ethanol extracts of peel, pulp and seeds of exotic Brazilian fruits. Food Res. Int. 2012, 49, 334-344. [CrossRef]

29. Kuroda, K.; Yagami, S.; Takama, R.; Fukushima, K. Distribution of coniferin in freeze-fixed stem of Ginkgo biloba L. by cryo-TOF-SIMS/SEM. Sci. Rep. 2016, 6, 31525. [CrossRef]

30. Demuth, H.; Jensen, S.R.; Nielsen, B.J. Iridoid glucosides from Asystasia bella. Phytochemistry 1989, 28, 3361-3364. [CrossRef] 
31. Wu, X.; Zhou, Y.; Yin, F.; Mao, C.; Li, L.; Cai, B.; Lu, T. Quality control and producing areas differentiation of Gardeniae Fructus for eight bioactive constituents by HPLC-DAD-ESI/MS. Phytomedicine 2014, 21, 551-559. [CrossRef] [PubMed]

32. Fu, Z.; Xue, R.; Li, Z.; Chen, M.; Sun, Z.; Hu, Y.; Huang, C. Fragmentation patterns study of iridoid glycosides in Fructus Gardeniae by HPLC-Q/TOF-MS/MS. Biomed. Chromatogr. 2014, 28, 1795-1807. [CrossRef] [PubMed]

33. Choze, R.; Delprete, P.G.; Lião, L.M. Chemotaxonomic significance of flavonoids, coumarins and triterpenes of Augusta longifolia (Spreng.) Rehder, Rubiaceae-Ixoroideae, with new insights about its systematic position within the family. Braz. J. Pharmacogn. 2010, 3, 20. [CrossRef]

34. Tauchen, J.; Bortl, L.; Huml, L.; Miksatkova, P.; Doskocil, I.; Marsik, P.; Villegas, P.P.P.; Flores, Y.B.; Damme, P.V.; Lojka, B.; et al. Phenolic composition, antioxidant and anti-proliferative activities of edible and medicinal plants from the Peruvian Amazon. Rev. Bras. Farmacogn. 2016, 26, 728-737. [CrossRef]

35. Andersen, W.K.; Omar, A.A.; Christensen, S.B. Isorhamnetin3-(2,6-dirhamnosylgalactoside)-7-rhamnoside and 3-(6-rhamnosylgalactoside)-7-rhamnoside from Rhazya stricta. Phytochemistry 1987, 26, 291-294. [CrossRef]

36. Zhu, X.; Zhang, H.; Lo, R. Phenolic Compounds from the Leaf Extract of Artichoke (Cynara scolymus L.) and Their Antimicrobial Activities. J. Agric. Food Chem. 2004, 52, 7272-7278. [CrossRef] [PubMed]

37. Brochado, C.D.O.; Almeida, A.P.; Barreto, B.P.; Costa, L.P.; Ribeiro, L.S.; Pereira, R.L.C.; Koatz, V.L.G.; Costa, S.S. Flavonol robinobiosides and rutinosides from Alternanthera brasiliana (Amaranthaceae) and their effects on lymphocyte proliferation in vitro. J. Braz. Chem. Soc. 2003, 14, 449-451. [CrossRef]

38. Brasseur, T.; Angenot, L. Six flavonol glycosides from leaves of Strychnos variabilis. Phytochemistry 1988, 27, 1487-1490. [CrossRef]

39. Buschi, C.A.; Pomilio, A.B. Isorhamnetin 3-O-robinobioside from Gomphrena martiana. J. Nat. Prod. 1982, 45, 557-559. [CrossRef]

40. Halim, A.F.; Saad, H.E.A.; Hashish, N.E. Flavonol glycosides from Nitraria retusa. Phytochemistry 1995, 40, 349-351. [CrossRef]

41. Hasan, A.; Ahmed, I.; Jay, M.; Voirin, B. Flavonoid glycosides and an anthraquinone from Rumex chalepensis. Phytochemistry 1995, 39, 1211-1213. [CrossRef]

42. Kaouadji, M. Flavonol diglycosides from Blackstonia perfoliata. Phytochemistry 1990, 29, 1345-1347. [CrossRef]

43. Hou, W.-C.; Lin, R.-D.; Lee, T.-H.; Huang, Y.-H.; Hsu, F.-L.; Lee, M.-H. The phenolic constituents and free radical scavenging activities of Gynura formosana Kiamnra. J. Sci. Food Agric. 2005, 85, 615-621. [CrossRef]

44. Gallegos-Olea, R.S.; Borges, M.O.R.; Borges, A.C.R.; Freire, S.M.F.; Silveira, L.M.S.; Vilegas, W.; Rodrigues, C.M.; Oliveira, A.V.; Costa, J.L. Flavonoides de Calotropis procera R. Br. (Asclepiadaceae). Rev. Bras. Plantas Med. 2008, 10, 29-33.

45. Tsiklauri, L.K.; An, G.; Alania, M.D.; Kemertelidze, E.P.; Morris, M.E. Optimum HPLC parameters for simultaneous determination of robinin and kaempferol. Pharm. Chem. J. 2012, 46, 64-67. [CrossRef]

46. Veitch, N.C.; Elliott, P.C.; Kite, G.C.; Lewis, G.P. Flavonoid glycosides of the black locust tree, Robinia pseudoacacia (Leguminosae). Phytochemistry 2010, 71, 479-486. [CrossRef] [PubMed]

47. Asres, K.; Eder, U.; Bucar, F. Studies on the anti-inflammatory activity of extracts and compounds from the leaves of Melilotus elegans. Ethiop. Pharm. J. 2000, 18, 15-23.

48. Ghareeb, M.A.; Shoeb, H.A.; Madkour, H.M.F.; Refahy, L.A.; Mohamed, M.A.; Saad, A.M. Antioxidant and cytotoxic activities of flavonoidal compounds from Gmelina arborea Roxb. Global J. Pharm. 2014, 8, 87-97. [CrossRef]

49. Boubaker, J.; Sghaier, M.B.; Skandrani, I.; Ghedira, K.; Chekir-Ghedira, L. Isorhamnetin 3-O-robinobioside from Nitraria retusa leaves enhance antioxidant and antigenotoxic activity in human chronic myelogenous leukemia cell line K562. BMC Complement. Altern. Med. 2012, 12, 135. [CrossRef] [PubMed]

Sample Availability: Samples of the compounds GAF 1, GAF 2, GAF 3, GAF 4 and GAF 5 are available from the authors.

(C) 2018 by the authors. Licensee MDPI, Basel, Switzerland. This article is an open access article distributed under the terms and conditions of the Creative Commons Attribution (CC BY) license (http://creativecommons.org/licenses/by/4.0/). 